\title{
Material flow in heterogeneous friction stir welding of thin aluminium sheets: Effect of shoulder geometry
}

\author{
R.M. Leal ${ }^{\mathrm{a}, \mathrm{b}}$, C. Leitão $^{\mathrm{a}}$, A. Loureiro $^{\mathrm{a}}$, D.M. Rodrigues $^{\mathrm{a}, *}{ }^{*}$ P. Vilaça ${ }^{\mathrm{c}}$ \\ a CEMUC, Department of Mechanical Engineering, University of Coimbra, Portugal \\ ${ }^{\mathrm{b}}$ ESAD.CR, Polytechnic Institute of Leiria, Caldas da Rainha, Portugal

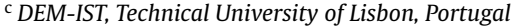

\section{A R T I C L E I N F O}

\section{Article history:}

Received 13 June 2008

Received in revised form 12 August 2008

Accepted 14 August 2008

\section{Keywords:}

Friction stir welding

Shoulder geometry

Material flow

Aluminium alloys

\begin{abstract}
A B S T R A C T
The aim of this investigation was to study the influence of tool geometry on material flow during heterogeneous friction stir welding in $1 \mathrm{~mm}$ thick plates of AA 5182-H111 and AA 6016-T4 aluminium alloys. Two types of tool shoulders were used: a shoulder with a conical cavity and a scrolled shoulder. Pin-driven flow was predominant in welds produced with the conical cavity shoulder, which are characterized by an onion ring structure. The interaction between pin-driven and shoulder-driven flow is restricted to the crown of the weld, at the trailing side of the tool, and extends throughout the weld thickness, at the leading side. Although no onion ring structure was formed in welds done with the scrolled shoulder, extensive mixing of the base materials occurred in a plasticized layer flowing through the thickness around the rotating pin. Shoulder-driven flow is intense and continuous around the tool.
\end{abstract}

(C) 2008 Elsevier B.V. All rights reserved.

\section{Introduction}

When compared with conventional fusion welding processes, the friction stir welding (FSW) process has many technical advantages for joining soft materials, such as aluminium alloys, beyond the evident environmental benefits. In fact, FSW is a solid state welding process that allows similar or dissimilar materials to be welded together without emission of radiation or dangerous fumes.

However, as for any other welding processes, FSW requires a careful choice of process parameters in order for microstructural and mechanical characteristics and defect free welds to be consistently reproducible. Defects in FS welds have already been classified as flow or geometric related [1]. The geometric related defects are usually associated with lack of penetration and occur due to insufficient pin penetration depth and/or improper seam tracking. The flow related defects, which are much more difficult to avoid, include flash formation, surface galling, lack of fill, wormholes, and nugget collapse or lack of consolidation [1-7].

According to the literature, the main process parameters influencing material flow and weld quality include tool geometry (pin and shoulder design and relative dimensions of pin and shoulder), welding parameters (tool rotation rate and direction, traverse speed, plunge depth, tilt angle), base material flow stress behaviour

\footnotetext{
* Corresponding author. Tel.: +351 239790 700; fax: +351 239790701

E-mail address: dulce.rodrigues@dem.uc.pt (D.M. Rodrigues).
}

and temperature as well as the interaction between the workpiece material and the various weld tool features [2,8-11]. Experimental flow visualization studies have been conducted by introducing marker materials into the weld line [9-15] by welding dissimilar materials $[14,16,17]$ or by simply using etching contrast to enhance the flow patterns in the weld [1,6,18-21]. However, all these techniques have well known limitations. The insertion of markers in the weld line may influence material flow during welding due to the different flow characteristics of the base and marker materials and the introduction of additional interfaces [22]. In the same way, it is not clear if the material flow during welding of dissimilar materials, having different flow properties, can be compared with material flow in friction stir welding of similar materials. Finally, all these techniques are limited to speculation about real material flow during FSW since they are based on the simple observation of pre- and post-welding material positions.

Despite current limitations of metal flow visualization techniques, it is commonly agreed that the flow of material around the tool is not symmetric about the weld centreline and displays significant differences between the advancing and retreating sides $[1,6,9-11,13]$. According to several authors, vertical, straightthrough and rotational flows of plasticized material take place in the vicinity or around the tool, dragging the bulk of the stirred material to a final position behind its original position $[6,11,13,14,20,23]$ In the wake of the weld, behind the travelling tool, material deposition takes place layer-by-layer resulting in the formation of a zone with a banded structure commonly referred to as the 
Table 1

Nominal chemical composition of the aluminium alloys, wt\% (Al-balance)

\begin{tabular}{|c|c|c|c|c|c|c|c|c|}
\hline Alloy & $\mathrm{Si}$ & $\mathrm{Fe}$ & $\mathrm{Cu}$ & $\mathrm{Mn}$ & $\mathrm{Mg}$ & $\mathrm{Cr}$ & $\mathrm{Zn}$ & $\mathrm{Ti}$ \\
\hline AA 5182-H111 & $<0.2$ & $<0.35$ & $<0.15$ & $0.2-0.5$ & $4.0-5.0$ & $<0.1$ & $<0.25$ & $<0.1$ \\
\hline AA 6016-T4 & $1.0-1.5$ & $<0.5$ & $<0.2$ & $<0.2$ & $0.25-0.6$ & $<0.1$ & $<0.2$ & $<0.15$ \\
\hline
\end{tabular}

Table 2

Tensile properties of the base materials

\begin{tabular}{lll}
\hline Alloy & $R_{\mathrm{p} 0.2}(\mathrm{MPa})$ & $R_{\mathrm{m}}(\mathrm{MPa})$ \\
\hline AA 5182-H111 & 108 & 350 \\
AA 6016-T4 & 104 & 260
\end{tabular}

$R_{\mathrm{p} 0.2}$-yield stress; $R_{\mathrm{m}}$-tensile strength.

nugget. Spacing between banding in the nugget is typically the same as the distance travelled by the tool during a single rotation [6,18-21,23-25].

With regard to the influence of the tool in the FSW flow field, two different modes of tool related flows are frequently mentioned in literature: shoulder-driven flow and pin-driven flow $[6,10,11,14,20,25-27]$. Although several studies concerning the influence of pin geometry in material flow during FSW have been carried out $[9,11,13,26]$, the influence of shoulder geometry on material flow is still unexplored. Nevertheless, a strong influence of shoulder geometry on the microstructure and mechanical behaviour of the FS welds has already been reported [28-30]. In addition, most of the research mentioned above was based on thicker materials, with thicknesses above $5 \mathrm{~mm}$, and only one paper, to the knowledge of the authors, examines FS welds less then $1 \mathrm{~mm}$ thick [31]. Even in this case, the subject of this study is microstructure and mechanical properties. In conclusion, although significant efforts have been made to investigate the FSW operating mechanism, material flow during welding remains largely uncharacterized, especially for very thin plates. In this investigation, material flow during FSW of thin plates, produced with different tool geometries and welding parameters, will be studied and analysed based on previous material flow theories.

\section{Experimental procedure}

$1 \mathrm{~mm}$ thick plates of two aluminium alloys currently used in the automotive industry, AA 5182-H111 and AA 6016-T4, were FS butt welded. The nominal chemical composition and mechanical properties of the sheets are depicted in Tables 1 and 2. A conventional milling machine provided with steel clamping and backing devices, to firmly fix the plates, was used to execute the welds. Welds were done with tool control position, and tool plunge force was not measured. The aluminium alloy AA 5182-H111 was always positioned to the advancing side of the tool.

Two series of butt welds were performed between both alloys using tools with different geometries and welding parameters. In the first series (WS1), a tool with a $10 \mathrm{~mm}$ diameter shoulder with an $8^{\circ}$ conical cavity and a left-threaded cylindrical probe of $3 \mathrm{~mm}$ in diameter and $0.9 \mathrm{~mm}$ in length was used (Fig. 1a). This tool was used with a $2^{\circ}$ tilt angle. In the second series (WS2), a tool with a

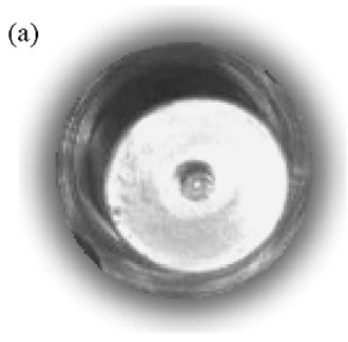

$5 \mathrm{~mm}$

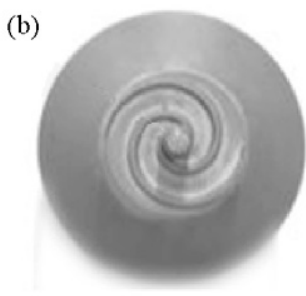

$\underline{7 \mathrm{~mm}}$
Fig. 1. (a) Conical shoulder and (b) scrolled shoulder.

Table 3

Welding parameters

\begin{tabular}{lllll}
\hline Series & $\begin{array}{l}\text { Rotation speed } \\
\omega(\mathrm{rpm})\end{array}$ & $\begin{array}{l}\text { Travel speed } v \\
(\mathrm{~mm} / \mathrm{min})\end{array}$ & $\begin{array}{l}\text { Tool tilt angle } \alpha \\
\left({ }^{\circ}\right)\end{array}$ & $\begin{array}{l}\text { Plunge depth } \\
(\mathrm{mm})\end{array}$ \\
\hline WS1 & 1800 & 160 & 2.5 & 0.95 \\
WS2 & 1120 & 320 & 0 & 0.95 \\
\hline
\end{tabular}

scrolled shoulder of $14 \mathrm{~mm}$ diameter and $0^{\circ}$ tilt angle was used (Fig. 1b). The probe was similar to that used with the previous tool. Several tests were performed to determine the set of welding parameters which gave good welds for each tool. Table 3 shows the welding parameters used in both series. Assuming a relation between the welding parameters and the energy input per unit of length of the weld $[13,24,32-34]$, it is possible to conclude that the welds of the first series, produced with lower tool traverse speed and higher tool rotation rate, were warmer than that of the second series.

Macroscopic and X-ray examination of the welds was performed prior to metallographic analysis in order to ensure that the welds were free of defects. Metallographic examinations were done in transverse, longitudinal and horizontal sections of the welds. The longitudinal cross-sections were taken at the weld axis and at different locations within the advancing and retreating sides of both types of welds. The horizontal cross-sections sampled several planes parallel to the weld surface at different distances from the root of the welds. The metallographic specimens were polished and etched with two different reagents: modified Poulton's and Hatch. The distribution across the weld of some specific chemical elements of each aluminium alloy, such as magnesium and silicon, was evaluated using an electron microprobe equipped with wavelength dispersive spectrometers.
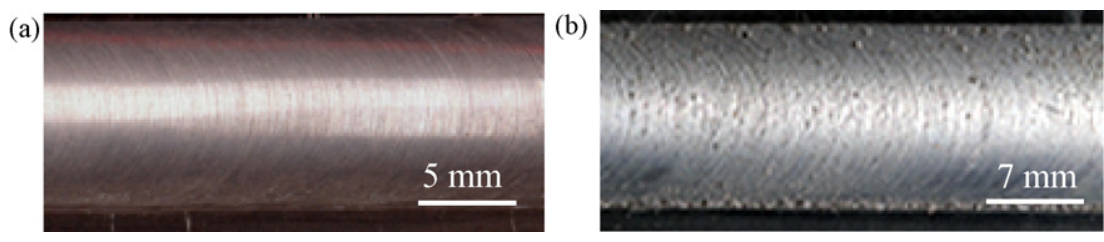

Fig. 2. Crown appearance of the WS1 (a) and WS2 (b) welds. 


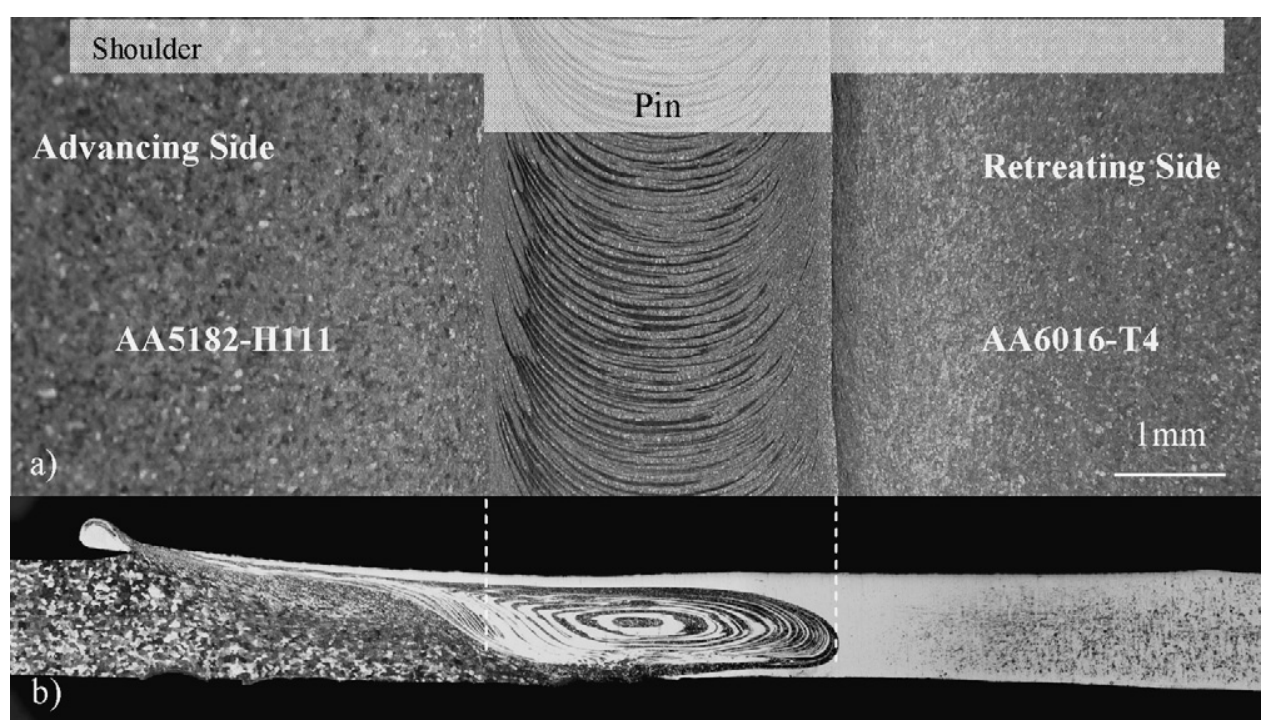

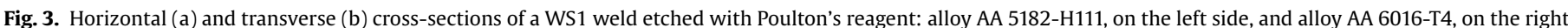
side.

\section{Results and discussion}

\subsection{First welding series (WS1)}

No defects were found in the X-ray analysis of the welds of the first series (WS1). The appearance of the WS1 weld crowns was very smooth, with regularly distributed arc-shaped striations and insignificant flash, as shown in Fig. 2a. As a result of tool tilt angle [35], there was a reduction in thickness relative to the base plate of around $0.06 \mathrm{~mm}$ in the middle of these welds.

Fig. 3 shows a horizontal section (Fig. 3a), at $0.25 \mathrm{~mm}$ from the weld root, and a transverse cross-section (Fig. 3b) of a WS1 weld. In order to facilitate the interpretation of the results, a sketch of the tool was drawn at the top of the picture. Both figures display the alloy AA 5182-H111, on the left side, and the alloy AA 6016T4, on the right side. Differential etching with Poulton's reagent enabled the distribution and mixing of both materials across the weld to be clearly seen. As can be observed in the transverse section, the nugget of the weld exhibits a classical onion ring structure composed of intercalated layers of both materials. In the horizontal cross-section the intercalated layers appear as very regularly spaced arc-shaped features. The spacing between the arcs is approximately $0.09 \mathrm{~mm}$, which corresponds to the distance the tool advances per revolution, as previously noted by several authors [6,18-21,23-25].

Fig. $4 \mathrm{a}$ and $\mathrm{b}$ shows longitudinal sections of these welds, including the final hole left by the probe at the end of the weld. It is important to emphasise that no stop action technique was used, thus it is possible that the macrostructure presented in the figure could result from more than a single revolution of the tool at the same traverse position. While the section in Fig. 4a illustrates the advancing side of the weld, at $0.3 \mathrm{~mm}$ from the weld axis, the section in Fig. $4 \mathrm{~b}$ corresponds to the retreating side of the weld sampled at the weld axis. In both images it is possible to distinguish a continuous white layer of AA 6016-T4 alloy behind the shoulder mark at the top of the weld, with intercalated arc shape features under it, which corresponds to the nugget zone.

In both images of Fig. 4 it is also possible to observe a spherical zone with an extremely fine-grained microstructure, surrounding the hole left by the pin, which corresponds to the pin-driven flow material. Ahead of the pin influence area, in Fig. 4b, it is also possible to observe a discontinuity corresponding to the initial interface between the faying surfaces of the plates, which has been bent by the shoulder towards the retreating side of the tool. This observation suggests that, ahead of the pin, the shoulder's influence area extends throughout the thickness, encompassing the pin influence area. Another aspect that supports this hypothesis is the presence of AA 5182-H111 material, between the crack and the pin influence area, which has been drawn from the advancing to retreating side by the tool shoulder. If Pictures $4 \mathrm{a}$ and $4 \mathrm{~b}$ are compared, it is evident that this through-thickness shoulder influence area is only visible ahead of the tool, extending to the retreating side, but for no more than $45^{\circ}$ relative to the weld axis, as shown in Fig. 5 . This picture shows views of four horizontal sections located at distances $t=0.7$,

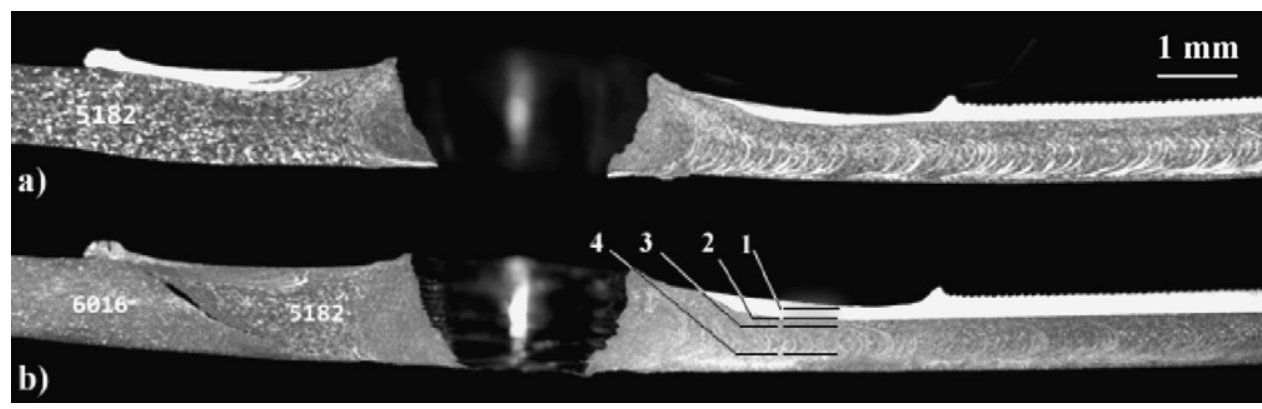

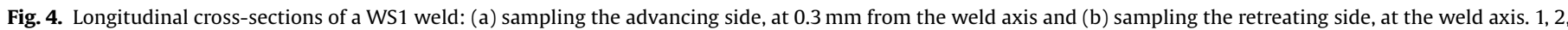
3 and 4 correspond to the location of the horizontal sections shown in Fig. 5. 

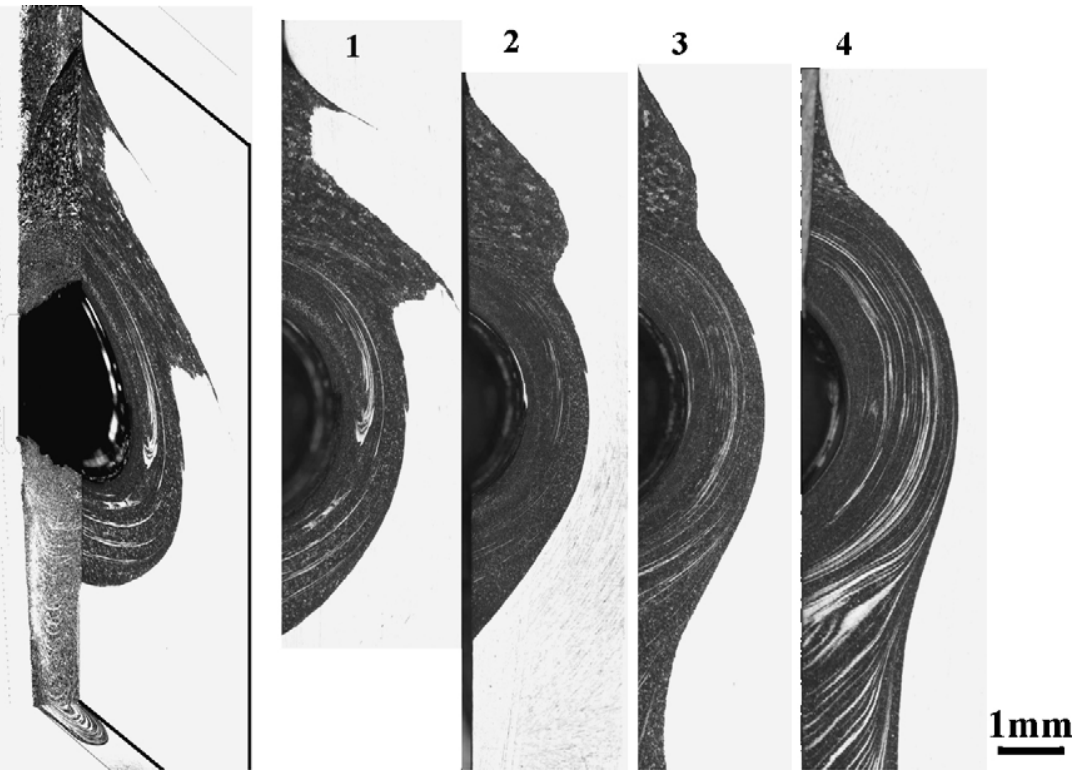

Fig. 5. Horizontal cross-sections of a WS1 weld at $0.7 \mathrm{~mm}(1), 0.58 \mathrm{~mm}(2), 0.48 \mathrm{~mm}(3)$ and $0.12 \mathrm{~mm}$ (4) from the weld root.

$0.58,0.48$ and $0.12 \mathrm{~mm}$ from the weld root (planes $1-4$ in Fig. $4 \mathrm{~b}$ ). In all these cross-sections, the portion of AA 5182-H111 material present in the shoulder influence area, in front of the tool, has the same grain structure as the unaffected base material. In the same pictures it is also possible to observe a thin layer of highly plastically deformed material surrounding the pin, which corresponds to the shear layer already observed by several authors $[10,14,15,19,20,36]$. At each tool rotation, layers of AA 5182-H11 material present in the shoulder influence area ahead of the pin enter the shear layer. According to previous publications, after one or more revolutions, the shear layer material is deposited, layer by layer, in the wake of the weld, producing the arc-shaped features observed in the longitudinal cross-sections of the welds. In Fig. 5 it is possible to observe material being transported from the shear layer into the weld seam. However, since no stop action technique was used in this investigation, it was not possible to observe the mechanism by which the layers are being detached. However, the authors believe that the deposition mechanism in these thin welds is not very different from that proposed by Chen et al. [20,25] for thicker plates.

From Figs. $4 \mathrm{~b}$ and 5 it is also possible to observe that, at the rear of the probe, where the successive layers of plasticized material are being continuously deposited, the shoulder influence area is restricted to the top surface of the weld, where it promotes the transport of the AA 6016-T4 alloy from the retreating to the advancing side of the tool. This transport of material at the top surface of the weld is visible in Figs. 3 and 4, where a white layer corresponding to the AA 6016-T4 alloy can be seen at the top of the weld, in both the transverse and longitudinal sections.

Assuming that FSW is a constant volume process [12,13,18], the portion of AA 6016-T4 material dragged by the shoulder is pushed downward within the pin diameter. During its downward movement around the pin, the AA 6016-T4 alloy is mixed with the AA 5182-H11 that is incorporated in the shear layer ahead of the tool, producing the intercalated onion ring structure presented in Fig. 3.

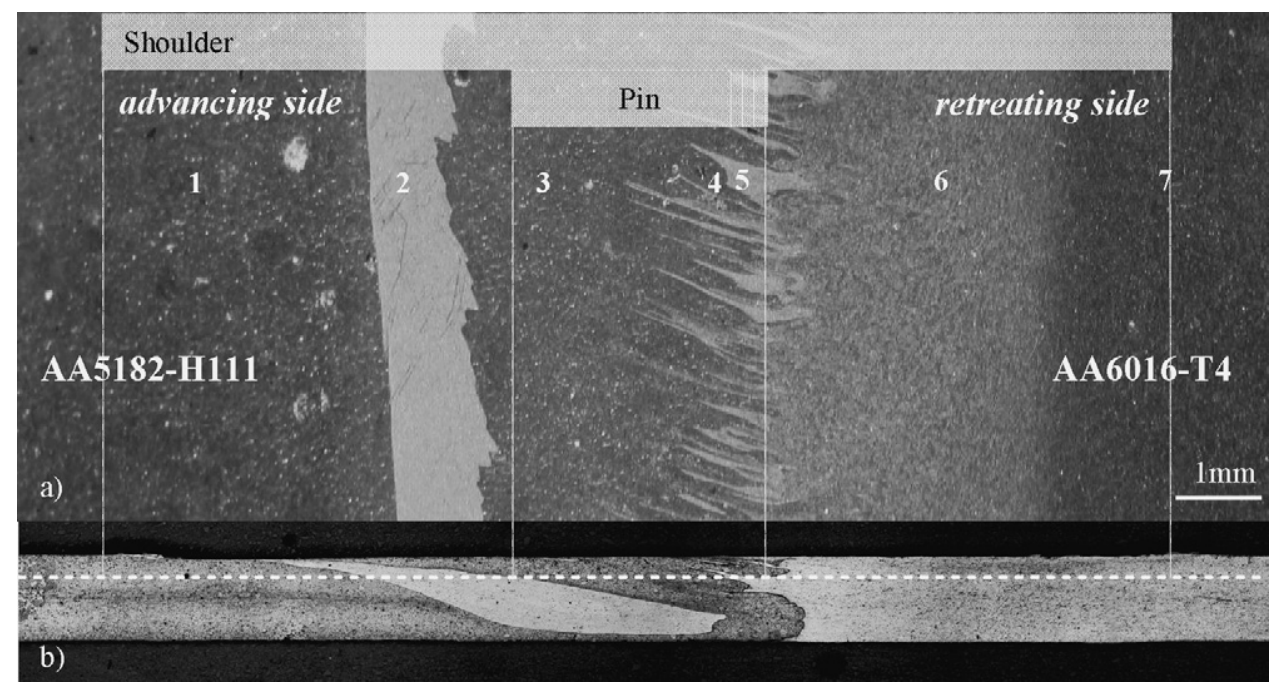

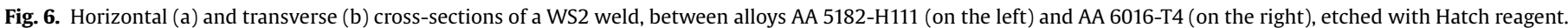
The horizontal dashed line indicates the through thickness position of the planar view in (a). 
Table 4

Chemical composition of several regions of a WS2 weld (wt\%)

\begin{tabular}{lrrlllllll}
\hline & 5182-H11 & 6016-T4 & \multicolumn{6}{l}{ Nugget zones } \\
\cline { 3 - 9 } & & & 1 & 2 & 3 & 4 & 5 & 6 & 7 \\
\hline $\mathrm{Mg}$ & $4-5$ & $0.25-0.6$ & 4.35 & 1.36 & 4.3 & 4.19 & 0.34 & 0.34 & 0.36 \\
$\mathrm{Si}$ & Max. 0.2 & $1-1.5$ & 0.05 & 0.74 & 0.05 & 0.04 & 0.84 & 0.81 & 0.79 \\
\hline
\end{tabular}

However, at the top of the weld, there is always some portion of AA 6016-T4 material that is not transported by the downward flow field and is expelled from the weld zone as flash at the advancing side of the tool (see Fig. 3), giving rise to the reduction in weld thickness relative to the base plate that was registered for these welds.

\subsection{Second welding series (WS2)}

The crown appearance of the welds of the second series (WS2) is shown in Fig. 2b. From this picture it is possible to observe that the crown of the WS2 welds is rougher than that of the welds of the first series. However, due to the absence of tool tilt angle, no reduction in thickness was observed for these welds.

Fig. 6 shows a horizontal section of a WS2 weld (Fig. 6a) and a transverse cross-section of the same weld (Fig. 6b). Both samples were etched with Hatch reagent, which enables the AA 5182-H111 alloy on the left side and the AA 6016-T4 alloy on the right side of the weld to be distinguished. As can be concluded by observing both pictures, no onion ring structure was formed in the pin influence area. In fact, as discussed below, material flow for these welds was very different from that of the welds of the first series.

From a purely metallographic analysis of Fig. 6, it is apparent that the materials in the weld nugget are mixed in a complex pattern with a tongue of white material going upwards through the advancing side into a matrix of AA 5182-H111 material. Since it is not possible to identify the nature of the materials in the different weld features from simple etching contrast, the metallographic study was complemented with a chemical analysis performed after a slight polishing in the plane parallel to the weld surface shown in Fig. 6a. As illustrated in this figure, seven different regions were analysed. Since both base materials are aluminium alloys, but with very different magnesium and silicon contents, these elements were selected to identify the materials present in the different zones under analysis.

Table 4 shows the chemical composition of the different regions identified in Fig. 6 together with the nominal content of magnesium and silicon of the parent materials. Comparing the results in the table, it is possible to confirm that zones 1 and 7 correspond, respectively, to the base materials AA 5182-H111 and AA 6016-T4. The magnesium and silicon contents of region 2 suggest that this zone is formed by a mixture of both materials. In the same way, it is possible to conclude that regions 3 and 4 are composed basically of the AA 5182-H111 alloy and regions 5 and 6 of the 6016-T4 alloy. This analysis suggests that the process induces some interpenetration of materials in the weld nugget but only a small amount of mixing.

Fig. 7 illustrates one transverse and two longitudinal views of a WS2 weld. In the transverse section in Fig. 7a, several cutting planes (1 -6) are indicated, which correspond to the location of the different sample sections presented in the following analysis. For example, the longitudinal view in Fig. $7 \mathrm{~b}$ shows a section sampled $0.6 \mathrm{~mm}$ from the weld axis, at the advancing side, that corresponds to plane 1 in Fig. 7a. In this figure, the flow patterns around the hole left by the pin suggest a strong interaction between the shoulderdriven and pin-driven material flows. This vast region of plasticized material, in which material flow lines propagate from the top to the bottom of the weld, is surrounded by well-defined bands of AA 5182-H111 material with a fine-grained microstructure. At the trailing side of the tool, next to the AA 5182-H111 fine-grained band, it is possible to observe two longitudinal layers of white and dark materials. If this is compared to Fig. 7a, where the cutting plane (1) corresponding to this sample is indicated, it is possible to conclude that the upper dark layer corresponds to zone 3 of the weld, and has a chemical composition similar to the AA 5182-H111 alloy, and the lower white layer corresponds to the mixed material in zone 2 .

Fig. 7c shows a longitudinal section, similar to that illustrated in Fig. 7b, but sampling the retreating side of the weld at the weld axis (plane 2 in Fig. 7a). As for the WS1 welds (Fig. 4b), in this picture it is also possible to observe a crack corresponding to the original faying surface between the plates, which was deflected by the tool to the retreating side. The location of the deflected interface corresponds to the outer border of the shoulder. Ahead of it is located the AA 6016-T4 retreating side material, and behind it, a portion of AA 5182-H111 alloy, drawn by the shoulder to the retreating side. Since the crack is almost perpendicular to the plate surface, and the material dragged from the advancing side has a fine-grained microstructure indicating intense plastic deformation, it is reasonable to assume that the stirring action of the shoulder extends throughout the plate's thickness. In order to understand the material flow in such a complex longitudinal pattern, four horizontal sections were analysed at $0.94,0.69,0.32$ and $0.21 \mathrm{~mm}$ from the weld root, as indicated in Fig. 7a (planes 3-6, respectively). Micrographs of the four slices were registered, after polishing and etching with modified Poulton's reagent, and are shown in Fig. 8a-d. The different flow zones around the tool were identified in all the horizontal sections by comparing Figs. 6 and 8 .

Analysing the slices corresponding to the horizontal sections at 0.32 and $0.2 \mathrm{~mm}$ from the bottom of the weld (Fig. $8 \mathrm{c}$ and $\mathrm{d}$ ), it is

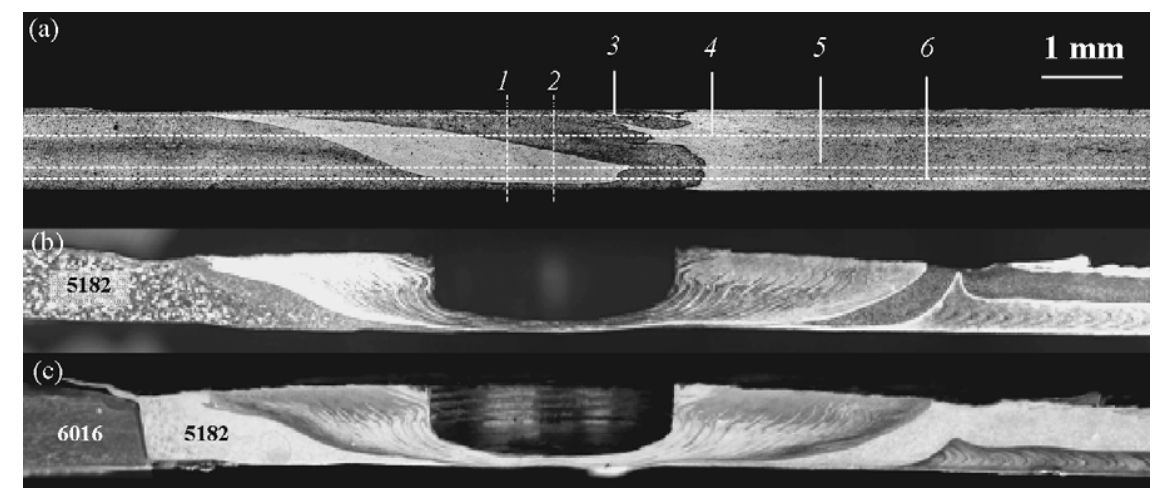

Fig. 7. Transverse (a) and longitudinal sections (b) and (c) of a WS2 weld; 3 to 6 represent the location of the horizontal planes shown in Fig. 8. 

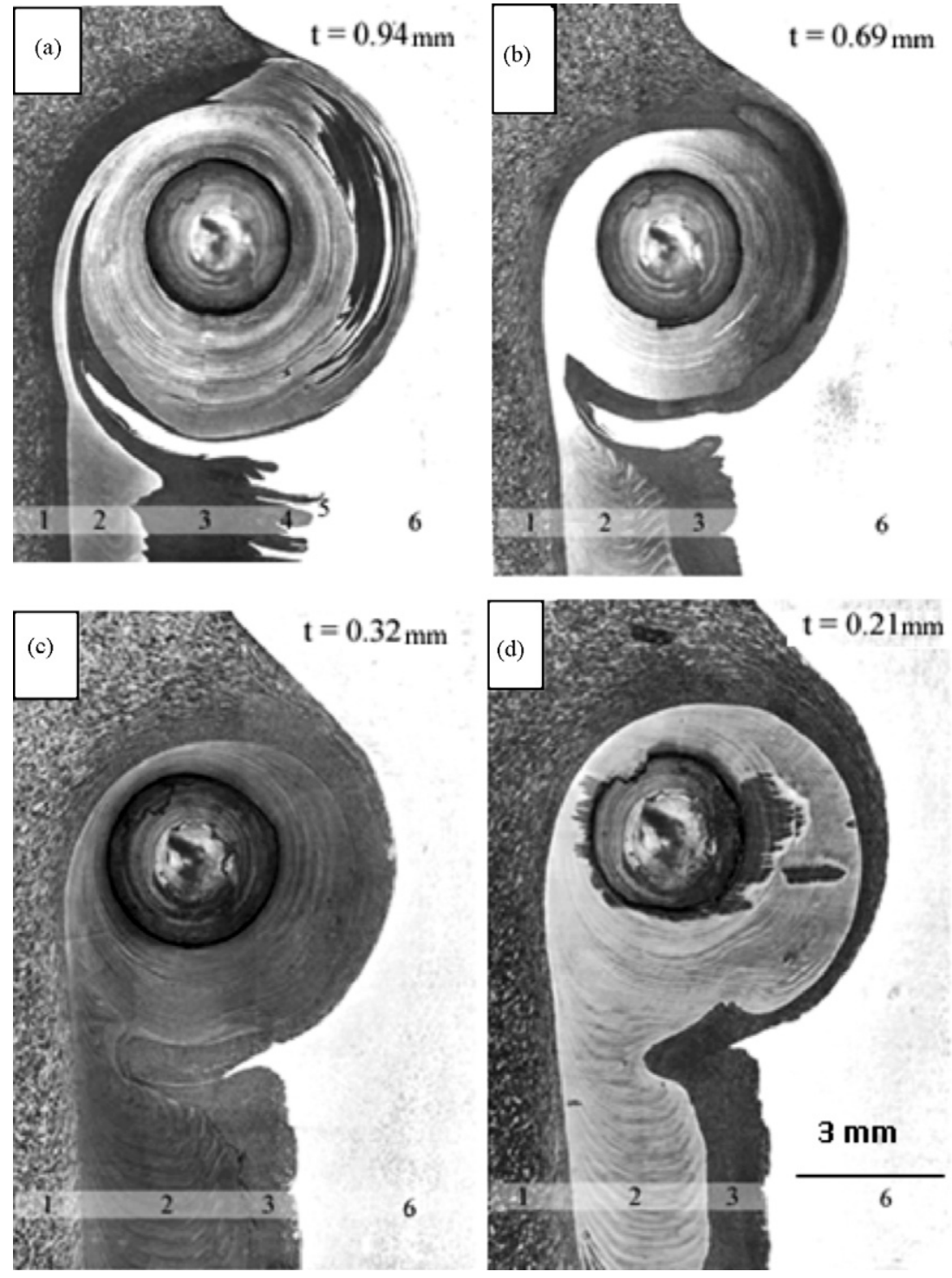

Fig. 8. Horizontal cross-sections of a WS2 weld at four different thicknesses $(t)$ from the weld root.

possible to observe in both pictures a layer of drastically plasticized material surrounding the hole left by the pin. This inner plasticized layer is surrounded by a layer of AA 5182-H111 material, which is drawn from the advancing side of the weld right around the tool, and is extruded against the inner shear layer at the back of the tool. Finally, it is possible to see that the retreating side material is drawn from the front to the rear of the tool almost parallel to the two inner flow layers. In fact, the AA 6016-T4 retreating side material is dragged into the inner flow only at the top of the weld, as shown in Fig. 8a and b, where it is possible to see a white strip of AA 6016-T4 alloy being pushed towards the advancing side of the weld, at the back of the tool.

In fact, in Fig. 8a it is clearly visible that, at the top of the weld, material from all the layers at the trailing side of the tool is pushed into the inner shear layer. These materials are mixed around the tool and flow from the top of the plasticized volume to the bottom (see flow patterns next to the pin hole in Fig. 7b and c), where it is sloughed off in the wake of the weld after one or more rotations, giving rise to the bottom mixed material layer observed in the longitudinal sections of the weld, at the rear of the tool influence area (Fig. $7 \mathrm{~b}$ and c). As already mentioned, this layer corresponds to the white tongue visible in the transverse sections of these welds. The elongated shape of the tongue, which extends towards the weld surface (see Fig. 7a), results from the stirring action of the shoulder pushing material from it, into the inner shear layer, at the top of the weld. At the bottom of the weld, the inner shear layer is constrained during its flow around the tool, by the AA 5182-H111 shear layer, which is also flowing around the tool. However, as it can be seen in Fig. 7b, c and d, the AA 5182-H111 layer does not complete an entire revolution, being extruded against the material deposited by the inner layer, at the trailing side of the tool. Finally, the third layer of the highly plasticized AA 6016-T4 material is extruded against the AA 5182-H111 layer.

\subsection{Discussion}

Comparing the results from previous analysis, it is possible to identify some similarities and also strong differences between the flow behaviour with the two types of tools. The main similarity is the presence of an inner shear layer of highly plasticized material surrounding the pin, for both types of tools (Figs. 5 and 8). After one or more revolutions, the material of these shear layers is sloughed 
of in the wake of the weld given rise to the entire weld, in the case of the welds of the first series, or to the mixed material layer (zone 2, Fig. 6), in the case of the welds of the second series. Another similarity between the material flows for the two types of tools is the transport of material by the shoulder, into the inner shear layer, at the top of the welds. More precisely, in the case of the welds of the first series, the retreating side material is dragged by the shoulder over the weld (Figs. 3 and 4) entering the inner shear layer by flowing downward within pin diameter. In the case of the welds performed with the scrolled shoulder, layers from all the weld zones at the rear of the tool are dragged by the shoulder, at the weld crown (Fig. 8a), into an inner flow that promotes its mixing and downward motion around the pin (Fig. 7a and b).

The main differences between the two flow mechanisms are associated with the deep differences in the shoulder-driven material flows around the tool. As it was shown in the previous analysis, for the tool with the conical cavity shoulder, the shoulder influence area is discontinuous and has different characteristics at the leading and trailing sides of the tool. In fact, at the trailing side of the tool, the shoulder influence area is restricted to the crown of the weld where it promotes the transference of material already described in previous paragraph. At the leading side of the tool, near the interface between the two plates, the shoulder influence area extends through the entire thickness of the plates dragging the AA 5182H111 alloy, from the advancing, to the retreating side of the tool in front of the pin (Figs. 4a and 5). This material is incorporated by the pin into the shear layer around it (Fig. 5) and is mixed with the AA 6016-T4 alloy that flows downward, from the shoulder influence area, with a circulating motion about the axis of the pin. Contrarily to this behaviour, for the tool with scrolled shoulder, it was possible to observe that the shoulder influence area extends through the entire thickness, all around the tool, with a straight interface with the surrounding base materials. The continuity of the shoulderdriven flow around the tool can be depicted from the continuous strip of highly plasticized advancing side material surrounding the tool (Figs. 7 and 8). At the trailing side of the tool, where the material from the shear layer is being continuously deposited against the advancing side material, this shoulder-driven material flow is disrupted, being extruded against the inner shear layer. However, the shoulder dragging action continues at the weld crown, moving material from the deposited layers into the inner shear layer, as previously described.

From previous analysis it is obvious that the amount of material moved from the advancing to the retreating side of the tool is much higher for the welds of the second series, and so, the

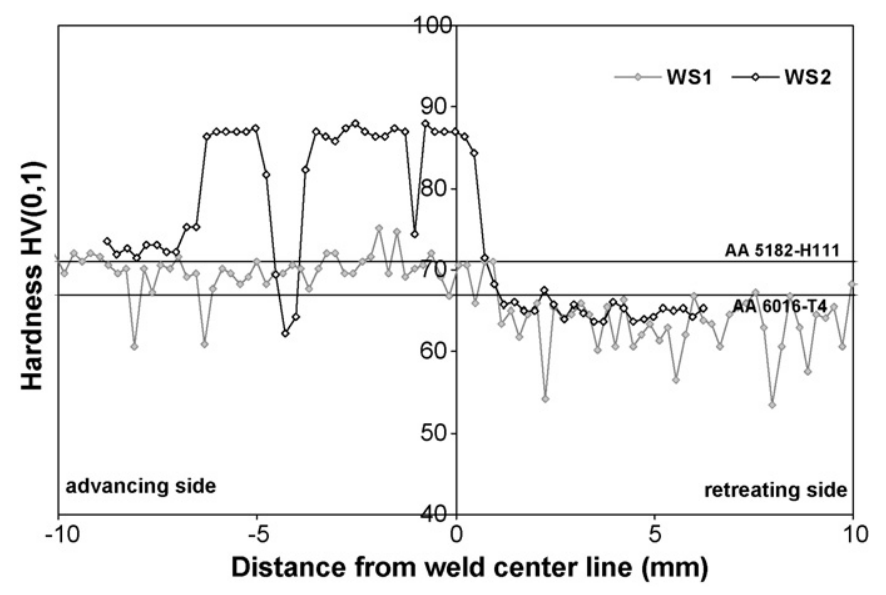

Fig. 9. Microhardness across the welds. amount of plastic deformation of the weld material. This has direct consequences in the mechanical behaviour of the welds. The heterogeneity in mechanical properties for both types of welds can be accessed from Fig. 9 where the microhardness profiles measured across both welds are plotted and compared. As it can be seen from this figure, the hardness profile across the WS1 weld is very regular with a smooth transition between the hardness values characteristic of the base materials. For the weld WS2, a strong increase in hardness is registered at the advancing side of the weld as a result of the intense plastic deformation of the hardenable AA 5182-H111 alloy. At the retreating side of the weld, the hardness values are close to that of the AA 6016-T4 base material. Naturally, this intense heterogeneity in mechanical properties determines the global plastic behaviour of the weld [37,38].

\section{Conclusions}

The effect of tool shoulder geometry on the material flow during heterogeneous friction stir welding of thin plates of two automotive aluminium alloys was investigated. A tool shoulder with a conical cavity was applied in the first weld series and a scrolled tool shoulder in the second series. The following conclusions were drawn:

- The first tool gives welds with excellent appearance but with some reduction in thickness. For these welds, the pin-driven flow is predominant in determining the characteristics of the weld nugget, yielding an onion ring structure caused by the flux of plasticized material around the pin and through plate thickness. The influence of the shoulder on the weld formation is also important, since it drags material into the shear layer that originates the weld nugget, through two different mechanisms acting simultaneously at the leading side of the weld and at the weld crown.

- Welds done with a scrolled shoulder tool do not display any reduction in thickness relative to the base plates and are less smooth than that of the first series. A substantial interaction between pin-driven and shoulder-driven material flow occurs in these welds, being the latter determinant for the weld structure. The amount of material moved from the advancing to the retreating side of the tool is much higher than that for the welds of the first series, and so, the amount of plastic deformation of the weld material, which yields strong heterogeneity in properties across the welds of the second series.

\section{Acknowledgements}

The authors are indebted to the Portuguese Foundation for the Science and Technology (FCT) and FEDER for the financial support through the POCI program and to Novelis Switzerland SA for supplying the aluminium sheets.

\section{References}

[1] W.J. Arbegast, Scripta Mater. 58 (2008) 372-376.

[2] Y.G. Kim, H. Fujii, T. Tsumura, T. Komazaki, K. Nakata, Mater. Sci. Eng. A 415 (2006) 250-254.

[3] H.-B. Chen, K. Yan, T. Lin, S.-B. Chen, C.-Y. Jiang, Y. Zhao, Mater. Sci. Eng. A 433 (2006) 64-69.

[4] K. Elangovan, V. Balasubramanian, Mater. Sci. Eng. A 459 (2007) 7-18.

[5] K. Kumar, S.V. Kailas, Mater. Design 29 (2008) 791-797.

[6] K. Kumar, S.V. Kailas, Mater. Sci. Eng. A 485 (2008) 367-374.

[7] V. Balasubramanian, Mater. Sci. Eng. A 480 (2008) 397-403.

[8] R.S. Mishra, M.Y. Ma, Mater. Sci. Eng. R 50 (2005) 1-78.

[9] L. Fratini, G. Buffa, D. Palmeri, J. Hua, R. Shivpuri, Sci. Technol. Weld. J. 11 (2006) $412-421$.

[10] H.N.B. Schmidt, T.L. Dickerson, J.H. Hattel, Acta Mater. 54 (2006) 1199-1209.

[11] Y.H. Zhao, S.B. Lin, F.X. Qu, L. Wu, Mater. Sci. Technol. 22 (2006) 45-50.

[12] K. Colligan, Supplement to the Weld. J. (July) (1999) 229s-237s.

[13] T.U. Seidel, A.P. Reynolds, Metall. Mater. Trans. A 32A (2001) 2879-2884. 
[14] M. Guerra, C. Schmidt, J.C. McClure, L.E. Murr, A.C. Nunes, Mater. Charact. 49 (2003) 95-101.

[15] J. Schneider, R. Beshears, J.A.C. Nunes, Mater. Sci. Eng. A 435-436 (2008) 297-304.

[16] Y. Li, L.E. Murr, J.C. McClure, Scripta Mater. 40 (1999) 1041-1046.

[17] Y. Li, L.E. Murr, J.C. McClure, Mater. Sci. Eng. A 271 (1999) 213-223.

[18] K.N. Krishnan, Mater. Sci. Eng. A 327 (2002) 241-251.

[19] J.A. Schneider, A.C. Nunes, J. Metall. Mater. Trans. B 35B (2004) 777-783.

[20] Z.W. Chen, T. Pasang, Y. Qi, Mater. Sci. Eng. A 474 (2008) 312-316.

[21] S. Xu, X. Deng, Acta Mater. 56 (2008) 1326-1341.

[22] H. Zhang, S.B. Lin, L. Wu, J.C. Feng, S.L. Ma, Mater. Design 27 (2006) 805-809.

[23] A.P. Reynolds, Scripta Mater. 58 (2008) 338-342.

[24] B. Yang, J. Yan, M.A. Sutton, A.P. Reynolds, Mater. Sci. Eng. A 364 (2004) 55-65.

[25] Z.W. Chen, S. Cui, Scripta Mater. 58 (2008) 417-420.

[26] S. Muthukumaran, S.K. Mukherjee, Sci. Technol. Weld. J. 11 (2006) 337-340.

[27] S. Muthukumaran, S.K. Mukherjee, Int. J. Adv. Manuf. Technol. 38 (2008) 68-73.
[28] A. Scialpi, L.A.C. De Filippis, P. Cavaliere, Mater. Design 28 (2007) 1124-1129.

[29] K. Elangovan, V. Balasubramanian, Mater. Design 29 (2008) 362-373.

[30] K. Kumar, S.V. Kailas, T.S. Srivatsan, Mater. Manuf. Process. 23 (2008) 188-194.

[31] A. Scialpi, M. De Giorgi, L.A.C. De Filippis, R. Nobile, F.W. Panella, Mater. Design 29 (2008) 928-936

[32] M.J. Peel, A. Steuwer, P.J. Withers, T. Dickerson, Q. Shi, H. Shercliff, Metall. Mater Trans. A 37A (2006) 2183-2193.

[33] A. Gerlich, P. Su, M. Yamamoto, T.H. North, J. Mater. Sci. 42 (2007) 5589-5601.

[34] H. Lombard, D.G. Hattingh, A. Steuwer, M.N. James, Eng. Fract. Mech. 75 (2008) 341-354

[35] A. Arici, S. Selale, Sci. Technol. Weld. J. 12 (2007) 536-539.

[36] R. Nandan, G.G. Roy, T.J. Lienert, T. Debroy, Acta Mater. 55 (2007) 883-895.

[37] C. Leitao, R.M. Leal, D.M. Rodrigues, A. Loureiro, P. Vilaça, Mater. Design 30 (2009) 101-108

[38] R.M. Leal, B.M. Chaparro, J.M. Antunes, P. Vilaça, D.M. Rodrigues, A. Loureiro, Mater. Sci. Forum 587-588 (2008) 961-965. 